\title{
65. A NEW ORBITAL CLASSIFICATION FOR PERIODIC COMETS
}

\author{
M. BIELICK I \\ Astronomical Observatory, Warsaw University, Warsaw, Poland
}

\begin{abstract}
A new classification for short-period comets is proposed - one based on minimum approach distances to the respective planets, rather than on aphelion distances.
\end{abstract}

Perturbations on the motion of a comet are of two general types:

(1) Slow variations during which time the comet does not pass close to a planet; and (2) the influences of close approaches to a planet.

The perturbations of the second type cause the cometary orbit to be drastically changed, and this can be considered the main factor in the evolution of the orbit over an interval of time comparable to the comet's lifetime. On the other hand, the present classification of periodic comets into planetary families according to their aphelion distances is quite artificial. Because of the inclinations of their orbits many cannot approach their supposed parent planets.

We therefore propose here a new definition, in which a comet is assigned to a particular family when the minimum distance between the orbits of the comet and corresponding planet is less than a certain value. The perturbations on a comet decrease very rapidly with increasing distance from a perturbing planet, the principal perturbing action during the close approach being of fairly short duration. If the above condition is fulfilled, we can consider that there exists a region, scarcely changing with time, where the cometary orbit is subject to large changes. The comet may repeatedly approach the planet in this region provided that

(1) The orbit of the comet remains elliptical after such an approach; and (2) no new region of approach is formed between the orbits of the comet and the same or some other planet.

In a particular interval of time a comet may develop one or more approach regions with one or more planets, or the comet may have no approach region. For the limiting distance for each planet we have adopted the radius of the sphere on which the perturbing acceleration due to the Sun is equal to the central acceleration in planetocentric motion (see Table I).

Considering the giant planets only the number of short-period comets in the various families now become: planetless, 21; Jupiter, 72; Saturn, 4; Saturn-Uranus, 1;

TABLE I

Limiting distances for planetary families (in AU)

\begin{tabular}{llllll}
\hline Mercury & 0.002 & Mars & 0.010 & Uranus & 0.67 \\
Venus & 0.010 & Jupiter & 0.50 & Neptune & 1.11 \\
Earth & 0.014 & Saturn & 0.62 & Pluto & 0.55 \\
\hline
\end{tabular}


Uranus, 1. The Saturn family consists of comets Wild, Peters, Herschel-Rigollet and Grigg-Mellish. The Saturn-Uranus family consists of $\mathrm{P} /$ Tempel-Tuttle, while the Uranus family contains $\mathrm{P} / \mathrm{de}$ Vico only. The Neptune family has no members. Many comets of the Jupiter family, such as d'Arrest, Kopff, Wolf, Borrelly, and Neujmin 2, were classified in the Jupiter family before; but some comets were classified in other families, as shown in Table II. It is interesting to note that the famous comets Encke and Halley both now belong to a planetless family.

TABLE II

Comet families according to the old and the new definitions

\begin{tabular}{llllrl}
\hline Comet & Old & New & Comet & Old & New \\
\hline Encke & J & O & Olbers & N & O \\
Tempel 2 & J & O & Herschel-Rigollet & $>$ P & S \\
Kulin & J & O & Pons-Brooks & N & O \\
Giacobini & J & O & Brorsen-Metcalf & N & J \\
Arend & J & O & Westphal & N & J \\
Gale & S & J & Oterma & S & J \\
Neujmin 1 & S-U & O & Lexell & $>$ P & J \\
Stephan-Oterma & U & O & Halley & N & O \\
\hline
\end{tabular}

$\mathrm{J}=$ Jupiter, $\mathrm{S}=$ Saturn, $\mathrm{U}=$ Uranus, $\mathrm{N}=$ Neptune, $\mathrm{P}=$ Pluto, and $\mathrm{O}=$ planetless.

TABLE III

Effect of orbital changes arising from large perturbations

\begin{tabular}{llll}
\hline Comet & Time interval & Old & New \\
\hline Schwassmann-Wachmann 2 & $1660-1730$ & S & J \\
& $1730-2060$ & J & J \\
Whipple & $1660-1775$ & S & J \\
& $1775-2060$ & J & J \\
Neujmin 3 & $1660-1850$ & S & J \\
Gale & $1850-2060$ & J & J J \\
& $1660-1917$ & S & J + S \\
Oterma & $1917-2060$ & S & J \\
& $1660-1937$ & S & J \\
Brooks 2 & $1937-1963$ & J & J \\
& $1963-2060$ & S & J \\
Lexell & $1660-1886$ & S & J \\
& 1886 & U & J \\
Kearns-Kwee & $1886-2060$ & J & J \\
& $1660-1767$ & J & $2 J$ \\
& $1767-1779$ & J & J \\
& $1779-2060$ & $>$ P & J \\
& $<1855$ & hyperbola & J \\
& $1855-1961$ & N & J \\
& $1961-2060$ & J & J \\
\hline
\end{tabular}


As a result of orbital evolution a comet can change from one family to another, but this was more frequent with the older classification (see Table III).

\section{Acknowledgments}

I should like to thank G. Sitarski for the use of his computer programme for calculating minimum distances and $\mathrm{K}$. Ziolkowski for his assistance. 